\title{
SENSITIVITY OF SKELETAL MUSCLE TO INTRA-ARTERIAL ACETYLCHOLINE IN NORMAL AND MYASTHENIC MAN
}

\author{
By GEORGE H. ACHESON, JOHN L. LANGOHR, AND JOHN B. STANBURY \\ (From the Department of Pharmacology, Harvard Medical School, and the Department of \\ Surgery of the Harvard Medical School at the Massachusetts General Hospital, Boston)
}

(Received for publication January 31, 1948)

The weakness and fatigability of skeletal muscle in myasthenia gravis have been likened to similar phenomena which occur in curare poisoning. As the intensity of curarization increases, muscle groups weaken in a certain order. The same muscle groups are often affected in the same order as myasthenia gravis progresses. The mechanism of death by interference with respiration is often strikingly similar in the two conditions. These facts have suggested that myasthenia gravis may result from the presence in the body of a curarelike substance. In favor of this hypothesis could be cited (1) the great sensitivity of myasthenic patients toward curare (1), and (2) evidence for the production of this kind of substance by the muscles of myasthenic patients during exercise $(2,3)$.

One of the effects of curare is to diminish or abolish the stimulating action of acetylcholine upon the neuromuscular endplate. In experimental animals, curarization produces simultaneously a decrease in the amplitude of the muscular twitch on stimulation of the motor nerve and a decrease in the twitch-like response to acetylcholine (4). If, as seems likely, this antagonism to acetylcholine is the factor responsible for the neuromuscular block of curare poisoning, then the phrase "curare-like" action implies the diminution or abolition of the stimulating effect of acetylcholine in skeletal muscle. If the hypothesis is correct that the weakness of myasthenia gravis is due to the presence of a curare-like substance, the myasthenic muscle should be less sensitive than normal to stimulation by acetylcholine. Two groups of investigators ( 5 to 8) have compared the effects of relatively large doses of intra-arterially injected acetylcholine upon skeletal muscle in normal and myasthenic man. Both drew the conclusion that myasthenic muscles are more sensitive to acetylcholine than normal muscles. The aim of the observations reported below was to check this conclusion by determining the response of normal and myasthenic muscles to threshold concentrations of acetylcholine.

The previous conclusions could not be confirmed; myasthenic muscles were not notably more or less sensitive to acetylcholine than normal muscles.

\section{METHODS}

The injections of acetylcholine ${ }^{1}$ were made through a 24 or 25 gauge needle into the radial artery at the wrist or the brachial artery just above the tendon of the biceps muscle. The acetylcholine was injected within one to three seconds usually in a volume of $0.2 \mathrm{cc}$. Each subject received a number of injections (from two to six) at intervals of 30 to 60 seconds. The acetylcholine was used within an hour after it had been dissolved in sterile physiological saline solution.

The forearm was fixed to a frame clamped to a table edge in a position comfortable to the subject. When injections were made into the brachial artery, the slightly flexed fingers pulled against a gauze band attached via a pulley to a lever. When injections were made into the radial artery, the supine hand was held dorsiflexed by a padded bar and the opposing motion of the thumb was recorded by attaching a band held by adhesive tape to the metacarpophalangeal joint via a pulley to the writing lever. Rubber bands from the lever to a fixed point gave a degree of tension to the fingers or thumb such that slight voluntary movements were readily recorded, whereas strong voluntary contractions gave wide excursions of the writing point. The inscription was upon smoked paper.

In order to determine whether or not the doses of acetylcholine which were injected affected the transmission of nerve impulses across the neuromuscular junction, maximal stimulation of the appropriate motor nerves was sometimes carried out. The stimuli were condenser discharges ( 0.1 microfarads discharging through the subject). A broad, moist contact to the forearm or upper arm served as the anode, and a restricted contact through cotton wet with saline solution was the cathode. When injections were made into the brachial artery and the flexors of the fingers and wrist were arranged to record, the stimulating cathode was placed over the ulnar nerve at the elbow. As the voltage of the stimulus was increased, the twitches increased in amplitude to a maxi-

\footnotetext{
1 Kindly supplied by Hoffmann-LaRoche, Inc., Nutley, New Jersey.
} 
mum and then decreased somewhat. The voltage of the stimulus which made the largest twitch was used in subsequent observations. When injections were to be made into the radial artery and the opposing muscle of the thumb was arranged to record, the cathode was placed over the median nerve in the wrist. As the voltage of the stimulus was increased the twitches of this muscle quickly reached a maximal height and suffered no decline at higher voltages. In the subsequent observations, 1.5 times the smallest voltage producing maximal twitches was used.

The subjects were asked to make no voluntary motions of the arm or hand except on command, and to describe their experiences after the completion of the observations.

Since the five myasthenic subjects showed only moderate weakness, it was possible to omit Neostigmine Bromide medication for 12 to 20 hours before the injections were made. After a period of this duration without Neostigmine, the cholinesterase activity of the plasma, as measured by the method of Ammon (9), was between 80 and 100 per cent of normal values in three of these patients. One patient received a subcutaneous injection of $0.6 \mathrm{mgm}$. of Atropine Sulfate shortly before the intra-arterial injections of acetylcholine were performed. Each myasthenic subject gave evidence in both history and physical examination of slight to moderate weakness and fatigability of the muscles of the forearm and hand, which diminished after Neostigmine. Subjects $\mathrm{E}$ and $\mathrm{H}$ of Table $\mathrm{I}$ exhibited less weakness of these muscles than the other three subjects.

\section{RESULTS}

As in previous observations of the effects of intra-arterial acetylcholine ( 5 to 8,10 to 12 ), no systemic effects of acetylcholine were noted. Except for the contractions of skeletal muscle to be noted below, the phenomena observed did not differ from those noted by previous investigators. Each successful injection produced a marked flush and sweating of the area supplied by the artery into which the injection was made. With the smallest doses the subject reported a sensation of warmth or heat spreading peripherally over this area. With larger doses this sensation was intensified and accompanied by a sensation of tingling or pinsand-needles. As the doses increased the warmth or heat acquired a painful quality still referable mainly to the skin. Added to these were the sensations of muscular tension accompanying the contractions of skeletal muscle to be described below. With the largest doses this tended to be painful. All these sensory phenomena disappeared within 15 to 30 seconds after each injection. The flush, however, gradually disappeared in a period of about five minutes.

\section{Myasthenic and normal subjects; brachial artery and flexors of the wrist and fingers}

Acetylcholine chloride was injected in a volume of 0.2 cc. except in one myasthenic subject, in whom the doses were injected in a volume of 1.0 cc. In each subject, a series of ascending doses was injected. The smallest dose used (40 $\mu$ g.) produced no contractions of skeletal muscles (three subjects). The next greater dose (125 $\mu$ g.) produced small contractions in three out of five normal subjects but failed to do so in two myasthenic subjects. Doses of 300 and $420 \mu \mathrm{g}$. of acetylcholine produced contractions in two other my-
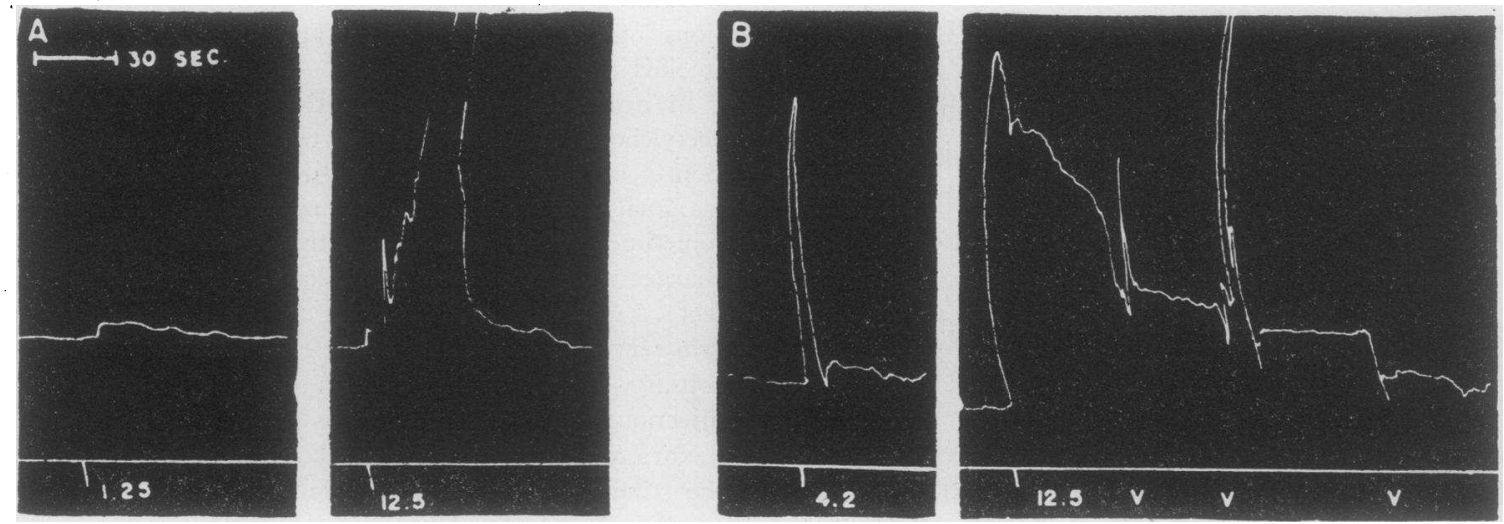

Fig. 1. Contractions of Flexor Muscles of Fingers and Wrist Produced by Rapid Injection of Acetylcholine into Brachial Artery

The dose in milligrams is indicated below the signal line. A. Normal subject (CB of Table I). B. Myasthenic subject ( $\mathrm{MK}$ of Table $\mathrm{I}$ ). V indicates voluntary contraction or relaxation. 
asthenic subjects. In a myasthenic subject 1.0 mgm. failed to produce contraction whereas 3 mgm. elicited a small contraction. In Table I, responses to the various doses injected in each of the ten subjects are listed in terms of the millimeters of excursion of the writing point of the myographic lever.

Figure 1 illustrates the contractions recorded in a normal (A) and a myasthenic (B) subject. The prolonged activity seen in the second tracing from the latter was also observed in normal subjects, and hence cannot be considered as characteristic of the myasthenic subjects. No qualitative or quantitative aspect of the contractions of the myasthenic subjects differed from those in the normal subjects.

\section{Discussion}

Previous investigators (5 to 8 ) alluded to an exquisite sensitivity of myasthenic muscles to acetylcholine as compared to normal. They interpreted this as indicating a compensatory sensitization of the muscles toward acetylcholine, resulting from the underlying cause of myasthenia gravis. An examination of the observations these investigators made, however, shows that no real basis existed for a statement about the sensitivity of normal and myasthenic subjects. Acetylcholine was injected into the brachial artery in doses of 10 to $50 \mathrm{mgm}$. (the largest dose of the present report was $12.5 \mathrm{mgm}$.). No attempt was made to find the smallest effective dose. In myasthenics these doses elicited strong contractions, whereas in normal subjects these large doses produced no contractions. The statements about sensitivity to acetylcholine were based upon the fact that these doses produced contraction in myasthenics but no contraction in normal subjects.

The observations reported here establish that when smaller doses of acetylcholine are injected intra-arterially in man, contractions of the skeletal muscles occur in both normal and myasthenic subjects. The aim of these observations was to determine whether or not there is a difference between the minimal dose of acetylcholine which stimulates the skeletal muscles in these two types of subjects. In the small groups studied, no significant difference either in threshold or in the character of the responses could be detected. Recent observations by Harvey, Lilienthal, and Talbot (13) confirm this conclusion.
Using much larger doses of acetylcholine (10 to $50 \mathrm{mgm}$.) the previous investigators ( 5 to 8 ) observed that strong contractions of the muscles of the hand and forearm occurred in myasthenic subjects, whereas no contraction occurred in normal subjects. This difference between normal and myasthenic subjects did not appear in the observations reported above, using doses from 0.04 to $12.5 \mathrm{mgm}$. of acetylcholine. It does not depend upon a difference of threshold of stimulation by acetylcholine. In a recent paper, Wilson and Stoner (14) reported that even with doses of $40 \mathrm{mgm}$. of acetylcholine no difference between normal and myasthenic subjects could be found.

Among the normal and myasthenic subjects, the thresholds to acetylcholine, as established by the procedure used, differed widely (see Table I).

TABLE I

Response by subjects in mm. excursion of myograms

\begin{tabular}{|c|c|c|c|c|c|c|c|c|c|c|}
\hline \multirow{2}{*}{$\begin{array}{c}\text { Dose in } \\
\text { mgm. }\end{array}$} & \multicolumn{5}{|c|}{ Normal } & \multicolumn{5}{|c|}{ Myasthenic } \\
\hline & GA & OK & $\mathbf{M}$ & O & CB & MK & D & $\mathrm{H}$ & $\mathbf{E}$ & $\mathbf{A B}$ \\
\hline $\begin{array}{l}0.042 \\
0.125 \\
0.3 \\
0.42 \\
1.0 \\
1.25 \\
3.0 \\
4.2 \\
5.0 \\
12.5\end{array}$ & $\begin{array}{l}\mathbf{0} \\
0\end{array}$ & 17 & $\begin{array}{l}0 \\
3\end{array}$ & 100 & 75 & $\begin{array}{l}60 \\
72\end{array}$ & $\begin{array}{l}10 \\
17\end{array}$ & 25 & $\begin{array}{r}6 \\
40 \\
42 \\
57\end{array}$ & $\begin{array}{l}0 \\
8\end{array}$ \\
\hline
\end{tabular}

Three normal subjects responded to $0.125 \mathrm{mgm}$.; another had but a slight response to ten times this dose. The myasthenic thresholds were distributed over a similar range. It seemed desirable to investigate this variability further. Additional series of observations were therefore carried out in normal subjects, as described in the succeeding section.

\section{Normal subjects: radial artery and opposing muscle of thumb}

In five normal subjects, an ascending series of doses of acetylcholine chloride was injected into the radial artery while the motor fibers to the opposing muscle of the thumb were being stimulated maximally once each 4.2 seconds. The smallest dose $(10 \mu \mathrm{g}$.) never produced muscular contraction or tingling, the next dose (30 $\mu \mathrm{g}$.) 
often produced a slight contraction of the opposer of the thumb accompanied by tingling sensations. In this series the succeeding doses (100 $\mu \mathrm{g} ., 300$ $\mu \mathrm{g}$., and $1 \mathrm{mgm}$. ) invariably produced contractions, usually of ascending amplitude and duration. In the series of observations to be reported in the next two paragraphs, some subjects had no response to $100 \mu \mathrm{g}$., but all who received $200 \mu \mathrm{g}$. exhibited muscular contractions.

Figure 2 illustrates responses to ascending doses of acetylcholine. The two tracings, $\mathrm{A}$ and $\mathrm{B}$, are from two subjects. The upper tracing shows brief contractions with all except the largest dose. The lower tracing illustrates the more prolonged contractions obtained in some subjects. No significant change in the amplitude of the twitches elicited by nerve stimulation occurred as a result of these injections of acetylcholine.

In a second series of subjects the same dose of acetylcholine chloride (30 or $100 \mu \mathrm{g}$.) was injected serially in different volumes of fluid (0.1, $0.2,0.4,1.0$, and $0.1 \mathrm{cc}$. or double these volumes). Whereas usually some muscular contraction oc- curred with one or another of these injections, the results were otherwise not predictable.

Hence a third series was undertaken in which the same dose of acetylcholine (100 or $200 \mu \mathrm{g}$.) was injected repeatedly in a volume of $0.2 \mathrm{cc}$. In eight subjects, a muscular response occurred with one or more of these injections. The magnitude of the responses and the intensity of the sensory phenomena accompanying these responses, however, varied considerably from one to another injection in the same subject. The type and degree of variation are illustrated in Figure 3.

\section{DISCUSSION}

These further observations in normal subjects confirm the variability in threshold to acetylcholine reported for normal and myasthenic subjects in Section I. Some subjects responded to $30 \mu \mathrm{g}$. and others needed $200 \mu \mathrm{g}$. of acetylcholine. These observations further indicate that even in single normal subjects there is considerable variation in the responses to the same dose of acetylcholine injected in the same volume of
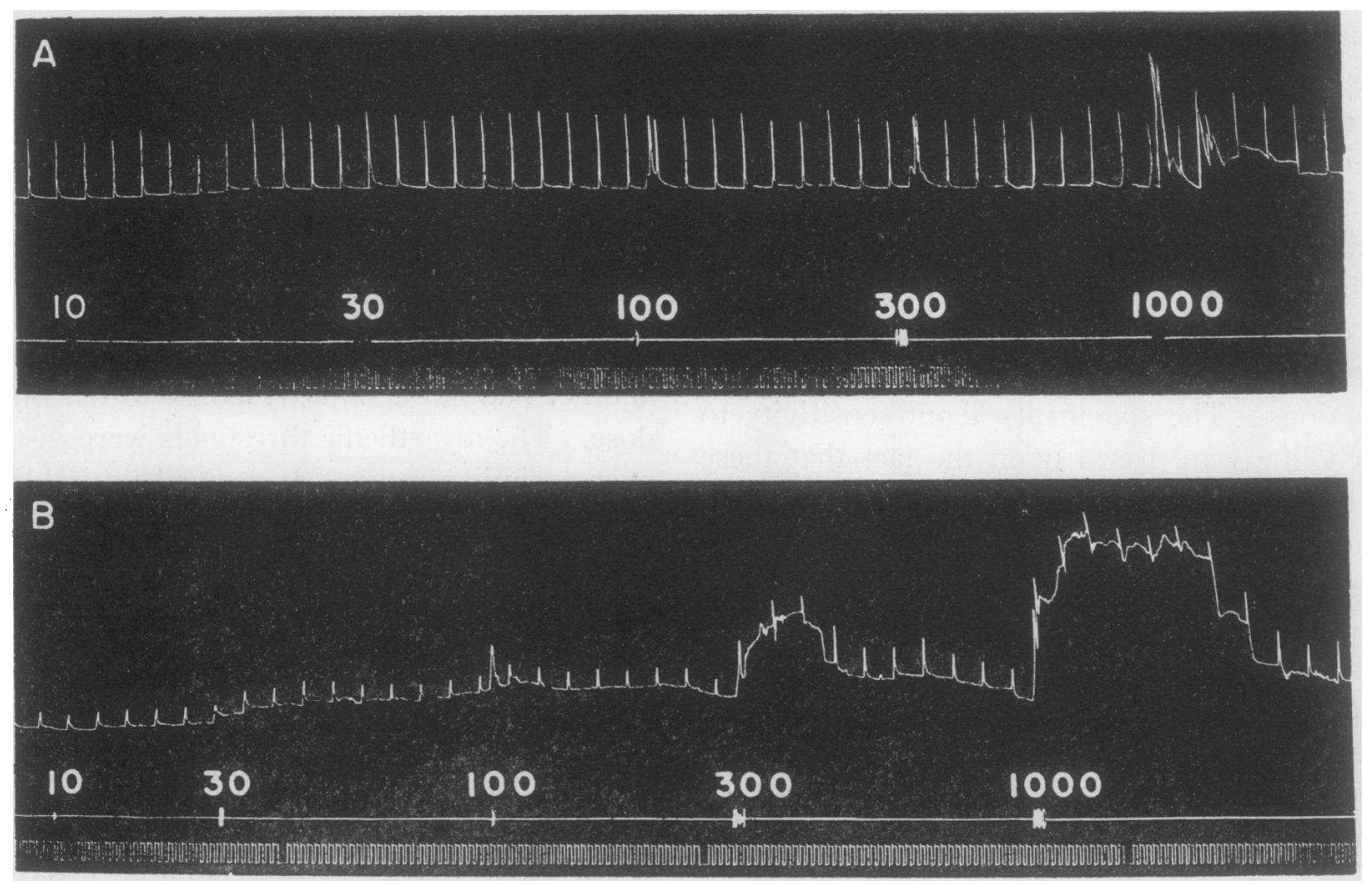

Fig. 2. Contractions of Opposing Muscle of the Thumb Produced by Rapid Injection of Acetylcholine into Radial Artery at Wrist

Throughout the period illustrated, there was maximal stimulation of the median nerve in the wrist every $4.2 \mathrm{sec}$. Doses are indicated in micrograms above the signal line. Bottom line: time in seconds and minutes. $\mathrm{A}$ and $\mathrm{B}$ : two normal subjects. 

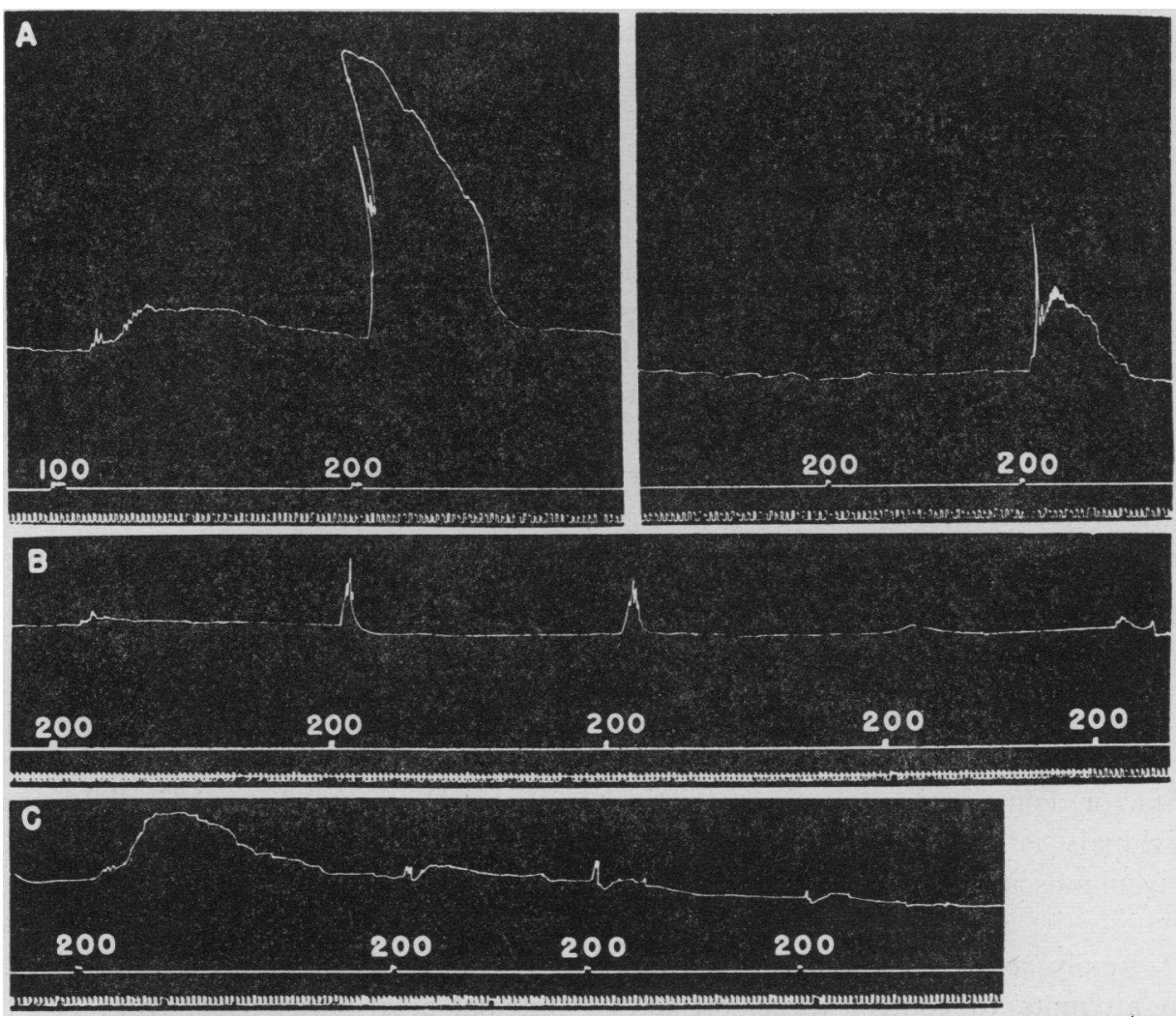

Fig. 3. Contractions of Opposing Muscle of the Thumb Produced by Rapid Injection of Acetylcholine into Radial Artery at Wrist

Doses are indicated in micrograms above the signal lint. A, B, and C: three normal subjects. Between the two parts of $A$, there was a pause of a little more than a minute for readjustment of needle in artery. Bottom line: time in seconds and minutes.

fluid at approximately the same rate when these injections are repeated at intervals of 30 to 60 seconds.

A. Sensitivity to acetylcholine in myasthenia gravis. These results emphasize the difficulty in establishing a threshold dose of acetylcholine for stimulation of skeletal muscle with accuracy. Since there is so much variation in normal subjects, data on a statistically significant number of subjects would be needed to establish within reasonable limits a normal threshold. Even this normal value would not be useful in judging the acetylcholine sensitivity of a single myasthenic patient; only a value derived from a large group of myasthenic patients would be comparable to the statistically established normal value.

These considerations suggest how difficult it would be with the methods used to determine whether or not myasthenia gravis results from a decrease in the sensitivity to acetylcholine. It seems likely in an all-or-none system like skeletal muscle that a slight change in sensitivity to the chemical mediator might lead to considerable weakness. A slight change in the threshold to acetylcholine would not be detectable by the methods thus far applied to the problem.

$B$. Variability of the response. What factors account for the variability of the response to the same dose of acetylcholine injected repeatedly into the same subject? The most difficult part of the manual technique of intra-arterial injection is the placing of the needle cleanly within the lumen of the artery and its maintenance in this position despite other necessary manipulations. But even when the manual technique was beyond reproach, striking differences occurred not only in the size of the motor response but also in the intensity of the sensory effects. There was no question of a 
systematic decrease in the sensitivity of the muscles; as illustrated in Figure 3, the early responses were not necessarily the greatest.

The possibility must be considered that the amount of acteylcholine reaching the muscles was not uniform from injection to injection. This might result partly from slight changes in the streamline flow of the injected fluid in the arteries. Probably more important is the fact that acetylcholine is a vasodilator. Even doses too small to elicit contractions of skeletal muscles produced intense flushing of the skin supplied by the artery into which the injection was made. This kind of vasodilatation is accompanied by very large increases of blood flow through the skin, especially in the fingers. It is possible that much of the drug which might have reached the muscle was diverted to the skin or was shunted away from both skin and muscle through arterio-venous anastomoses. A further factor contributing to the variation among individuals is the variability of arterial supply to given muscular masses, particularly in the hand, where the volar arch permits a wide variation of supply as between radial or ulnar artery. No attempts to control these variables were made in the observations reported here.

C. Stimulation of skeletal muscle by acetylcholine. Brown, Dale, and Feldberg (4) •established the fact that acetylcholine is capable of eliciting brief, twitch-like responses of skeletal muscle in experimental animals. In obtaining these responses, intravenous injection of acetylcholine was found to be quite ineffective, and even the injection into large arteries was an unreliable method. The best results were achieved by the injection of the acetylcholine into a relatively small artery near the muscle, occluding the artery proximal to the point of injection so that the injected substance would reach the muscle abruptly and relatively undiluted. This method the authors designated "close intraarterial injection." In the experimental preparation which they used, certain volumes of injected fluid and certain rates of injection were optimal for the production of a twitch with the smallest dose of acetylcholine.

In general outline, the methods used here in man resembled the "close intra-arterial injection" used in experimental animals. Of the two arteries into which the injections were made in man, the radial is the smaller and the closer to the muscle from which the records were made. The dimensions of the opposing muscle of the thumb are similar to those of the gastrocnemius muscle in the cat. Yet the radial artery supplies a considerable and variable part of the blood circulating the hand, and hence the acetylcholine is injected into larger and less uniform quantities of blood and vascular bed than is the case with the method of Brown, Dale, and Feldberg. When the injection is made into the brachial artery, the blood flow is even larger and the muscles studied have considerably greater mass and are more distant from the point of injection than with the radial artery. In the experiments reported here no occlusion of the artery was made during or after the injection.

The doses necessary to elicit these responses in man correspond to the probable conditions of blood flow, muscular mass, and distance of the muscle from the point of injection which were discussed above. Brown, Dale, and Feldberg found that 2.5 $\mu \mathrm{g}$. of acetylcholine were necessary to produce a small twitch-like response in the cat's gastrocnemius. When the radial artery was used in man, 30 or more $\mu \mathrm{g}$. were effective; and when the brachial artery was used, from 100 to $1000 \mu \mathrm{g}$. produced small responses in different subjects. Previous investigators injecting acetylcholine rapidly into the femoral artery in the groin found no muscular contraction with ten times these doses $(11,12)$.

The contractions of skeletal muscles obtained in man on the whole resemble those found by Brown, Dale, and Feldberg in the cat. Some of the responses reported by these authors were almost as brief as the single twitch. In our experiments none of the responses to acetylcholine were so brief. Many of those observed in man, however, closely resemble the responses to acetylcholine illustrated in Figure 2 of the paper by Brown, Dale, and Feldberg. As in the cat, the twitches elicited by stimulation of the motor nerve were unaffected by these injections (Figure 2). As the dose of acetylcholine was increased, the duration of the muscular responses in man tended to increase until they were definitely more prolonged than those observed by Brown, Dale, and Feldberg.

When the myographic tracing indicated a prolonged contraction (see Figures 1, 2, and 3) it is not certain that all of this response was due to the action of the injected acetylcholine on the muscle 
fibers. Often the tension of these muscles remained elevated for 15 to 45 seconds after the sensations which immediately followed the injection had disappeared. In some of these instances the subject felt that the muscle had relaxed to its original state while the myogram still indicated contraction. Since the sensory and motor connections of these muscles with the central nervous system were intact, it is possible that these prolonged contractions represented reflex changes of the tonic discharge of nerve impulses to these muscles or their antagonists. In Figure $1 \mathrm{~B}$ it may be noted that the interposition of voluntary contractions or relaxations changed the succeeding basal tension of the muscles.

\section{SUM MARY}

1. Acetylcholine rapidly injected into the brachial artery at the elbow elicits contractions in the muscles of the forearm and hand. The threshold doses range between 0.1 and $1.0 \mathrm{mgm}$.

2. Five patients with myasthenia gravis did not differ from five normal subjects with respect to the character of these contractions or the range of threshold doses of acetylcholine.

3. Acetylcholine rapidly injected into the radial artery at the wrist elicits contractions of the opposing muscle of the thumb. The threshold doses range between 30 and $200 \mu \mathrm{g}$.

4. Considerable variation of the muscular response occurs when the same dose is injected repeatedly at intervals of 30 to 60 seconds.

\section{BIBLIOGRAPHY}

1. Bennett, A. E., and Cash, P. T., Myasthenia gravis; curare sensitivity; a new diagnostic test and approach to causation. Arch. Neurol. \& Psychiat., 1943, 49, 537.
2. Walker, M. B., Myasthenia gravis: a case in which fatigue of the forearm muscles could induce paralysis of the extraocular muscles. Proc. Roy. Soc. Med., 1938, 31, 722.

3. Wilson, A., and Stoner, H. B., Myasthenia gravis : a consideration of its causation in a study of 14 cases. Quart. J. Med., 1944, 13, 1.

4. Brown, G. L., Dale, H. H., and Feldberg, W., Reactions of the normal mammalian muscle to acetylcholine and to eserine. J. Physiol., 1936, 87, 394.

5. Lanari, A., "Myasténia gravis" y transmisión química neuro-humoral. Rev. Soc. argent. de biol., 1937, 13, 239.

6. Harvey, A. M., Lilienthal, J. L., Jr., and Talbot, S. A., On the effects of the intra-arterial injection of acetylcholine and prostigmine in normal man. Bull. Johns Hopkins Hosp., 1941, 69, 529.

7. Harvey, A. M., and Lilienthal, J. L., Jr., Observations on the nature of myasthenia gravis. The intra-arterial injection of acetylcholine, prostigmine, and adrenaline. Bull. Johns Hopkins Hosp., 1941, 69, 566.

8. Harvey, A. M., Lilienthal, J. L., Jr., and Talbot, A. S., Observations on the nature of myasthenia gravis. The effect of thymectomy on neuromuscular transmission. J. Clin. Invest., 1942, 21, 579.

9. Ammon, R., Die fermentative Spaltung des Acetylcholins. Arch. f. d. ges. Physiol., 1934, 233, 486.

10. Ellis, L. B., and Weiss, S., A study of the cardiovascular responses in man to the intravenous and intra-arterial injection of acetylcholine. J. Pharmacol. \& Exper. Therap., 1932, 44, 235.

11. Carmichael, E. A., and Fraser, F. R., The effects of acetylcholine in man. Heart, 1933, 16, 263.

12. Fraser, F. R., MacGeorge, M., and Murphy, J. E., The action of choline esters in myasthenia gravis. Clinical Science, 1937, 3, 77.

13. Harvey, A. M., Lilienthal, J. L., Jr., and Talbot, S. A., Personal communication.

14. Wilson, A., and Stoner, H. B., The effect of the injection of acetylcholine into the brachial artery of normal subjects and patients with myasthenia gravis. Quart. J. Med., 1947, 16, 237. 